\title{
Challenge of optimising medication in people with severe mental illness
}

\author{
Ian Maidment (D) ,' Dolly Sud, ${ }^{1,2}$ Carolyn Chew-Graham ${ }^{3,4}$
}

${ }^{1}$ School of Pharmacy, College of Health and Life Sciences, Aston University, Birmingham, UK ${ }^{2}$ Pharmacy, Leicestershire Partnership NHS Trust, Leicester, UK

${ }^{3}$ School of Medicine, Keele University Institute for Primary Care and Health Sciences, Keele, UK

${ }^{4}$ West Midlands Applied Research Collaboration, West Midlands, UK

\section{Correspondence to}

Dr lan Maidment, School of Pharmacy, College of Health and Life Sciences, Aston University, Birmingham, B4 7ET, UK; i.maidment@aston.ac.uk

Accepted 16 September 2021

\section{SLinked}

http://dx.doi.org/10.1136/ bmjqs-2021-013427

\section{Check for updates}

(c) Author(s) (or their employer(s)) 2021. No commercial re-use. See rights and permissions. Published by BMJ.

To cite: Maidment I, Sud $D_{\text {, }}$ Chew-Graham C.

BMJ Qual Saf Epub ahead of print: [please include Day

Month Year]. doi:10.1136/

bmjgs-2021-013847
Mental health problems affect at least 1 in 4 people, cost the UK $£ 105$ billion annually and represent $28 \%$ of the total UK disease burden affecting all levels of society. ${ }^{1}$ Internationally, mental health accounts for 1 in 5 years lived with disability, and in 2019 WHO launched a special initiative specifically focused on mental health. ${ }^{2}$ COVID-19 is widely acknowledged to have increased this burden; early research identified a reduction in reported problems, but this most likely simply reflects the difficulty in accessing healthcare because many nonCOVID services had limited capacity. ${ }^{3}$ Primary care is key to identifying and managing people with mental health problems; data from England suggest that 40\% of general practitioner (GP) consultations involve mental health problems, and 30\% to $50 \%$ of people with severe mental illness (SMI) such as psychosis or bipolar disorder are solely supported by primary care without specialist input. ${ }^{4}$ Internationally, the WHO special initiative also identified the importance of primary care. ${ }^{2}$

Medication to reduce symptoms is one of the main treatments for people with SMI, who are frequently prescribed complex regimens including medication for both mental and physical conditions. However, such medications have potentially significant adverse consequences. For instance, antipsychotics are associated with an increased prevalence of cardiometabolic risk, metabolic syndrome and related diseases including dyslipidaemia, impaired glucose tolerance and weight gain and cardiovascular disease, which can lead to 'cascade prescribing' as other medications are then needed to manage these effects. ${ }^{5}$ Such cascade prescribing may not only be associated with adverse events including additional physical health problems, but such complex regimens are also more difficult for patients to manage. ${ }^{6}$ Prescribing medication for people with SMI should therefore be coupled with monitoring of the physical health problems that may occur.

Other factors also increase the frequency of physical health problems in people with SMI: lifestyle and behaviour (such as smoking), inequitable provision of healthcare despite greater need, different patterns of healthcare utilisation, and lack of integration among services provided by primary and secondary care. ${ }^{7}$ Rates of physical health monitoring in primary care are significantly lower for people with SMI than for the general population, despite consultation rates being much higher, ${ }^{89}$ which suggests that diagnostic overshadowing plays a significant role. Primary care therefore has a key role in safe prescribing of medication for people with SMI.

\section{SAFETY OF MENTAL HEALTH- RELATED PRESCRIBING IN PRIMARY CARE}

In this issue of BMJ Quality and Safety, Dr Khawagi and colleagues evaluate the safety of mental health related prescribing in general practice in the UK. ${ }^{10}$ They report on prevalence and between-practice variation in 22 indicators of mental healthrelated prescribing that suggest potentially hazardous prescribing $(n=18)$ or inadequate monitoring of medication $(n=4)$. The indicators had been developed in a previous Delphi study ${ }^{11}$ to target safety and monitoring of medication used to treat SMI, anxiety, insomnia, depression and dementia. In total, $90.2 \%$ and $9.4 \%$ of patients 'at risk' triggered at least one potentially inadequate medication monitoring event and one potentially hazardous prescription, respectively.

The study of Khawagi and colleagues builds on research over many years that has highlighted a range of issues with medication optimisation in mental health, ${ }^{16}{ }^{12}$ where medication optimisation is "a person-centred approach to 
Table 1 Examples of safety and monitoring Indicators ${ }^{10}$

Prescribing Safety Risperidone prescribed to a patient with dementia and without Indicator $2 \quad$ psychotic illness, for more than 6 weeks

Monitoring Prescribing lithium without monitoring lithium plasma levels

Indicator $3 \quad$ within the previous 6 months, or within the last 3 months if the patient is (1) aged $\geq 65$ years, (2) has a diagnosis of renal impairment or (3) is in their first year of treatment

safe and effective medicines use, to ensure the best possible outcomes". ${ }^{13}$ However, it is the first study to specifically assess the safety of mental health-related prescribing within primary care, using a suite of Prescribing Safety Indicators (see table 1 for examples) developed by a panel of mental health expert clinicians. Furthermore, even after controlling for differences in patient characteristics, they also found significant variation between practices for some indicators, for example, relating to benzodiazepine and Z-drug (eg, zopiclone) prescribing, and physical health monitoring in patients on antipsychotics.

Dr Khawagi and colleagues highlight some of the difficulties with very explicit prescribing indicators, both their indicators and others such as STOPP/START (Screening Tool of Older Persons' potentially inappropriate Prescriptions/Screening Tool to Alert to Right Treatment). ${ }^{14}$ Similar to clinical guidelines, these indicators may not fully capture the complexity of individual patients. Put another way, prescribing risperidone for more than 6 weeks might be appropriate if it prevents the patient from harming themselves or others. Thus, the presence of an indicator for potentially inappropriate prescribing, like the failure to exactly follow a clinical guideline, does not necessarily indicate an error. In those cases, what may on the face of it be seen as inappropriate prescribing might be in the patient's best interest and reduce the risk of harm. To judge whether a prescription is actually inappropriate therefore requires consideration of the individual situation, weighing up the risks and benefits in that individual.

\section{FACTORS DETERMINING THE RISK AND COMPLEXITY OF MEDICATION-RELATED ADVERSE EVENTS}

Multiple clinical and structural factors potentially increase the risk of medication-related adverse events in people with SMI. ${ }^{6}$ Polypharmacy continues to be common; one recent study found that $23.1 \%$ of adults with SMI taking antipsychotics were on more than one antipsychotic. ${ }^{15}$ Another study found that more than one type of psychotropic was prescribed for 31\% of pregnant women with psychotic disorders and for $30 \%$ with bipolar disorder. ${ }^{16}$

Medication and polypharmacy can have profound consequences for patients. A recent up-to-date review concluded that type 2 diabetes occurs in $12.8 \%$ of those on antipsychotics compared with $2.1 \%$ in untreated people with early schizophrenia, with the greatest relative risk of diabetes in those aged under $24{ }^{17}$ One recent cohort study in 99 older people with
SMI reported fatigue (67.7\%), dry mouth (66.6\%), obesity (27.3\%) and physical inactivity (57.6\%), all of which are potentially medication related. ${ }^{18}$ Studies have also reported that among patients diagnosed with diabetes, those with SMI have 50\% higher mortality ${ }^{19}$ and an increased risk of complications requiring specialist treatment ${ }^{20}$ compared with people without SMI. Other side effects include sedation and anticholinergic effects, which can result in cognitive impairment. ${ }^{21}$ These will add to the physical problems and complexity of mental health-related prescribing.

Members of ethnic minority communities are at a greater risk of both mental ill health and physical health problems such as diabetes. However, patients from ethnic minority communities also experience a wide range of inequality in service provision. They are less likely to be offered a full range of evidence-based interventions and treatments-including talking-based therapies and medication such as clozapine (the most effective medication for schizophrenia). ${ }^{22}$

\section{MOVING FORWARDS}

Primary care is central, with GPs and their teams, including pharmacists, at the heart in countries such as the UK, to delivering high-quality mental health care. ${ }^{23}$ A team approach working across primary, secondary and social care, in partnership with patients and family carers, is required to achieve medication optimisation with the skills of each team member fully utilised. Prescribing decision-making needs to actively involve patients and their family carers, explaining the need for and management of medication-to support people take prescribed medication. A holistic approach to care, including the use of medication, that considers cultural factors is vital to improving health outcomes in ethnic minority communities. ${ }^{22}$

Care also needs to be co-ordinated, as medications may be prescribed by different providers and GPs have a key and critical role in co-ordinating care. ${ }^{24}$ In terms of medication optimisation, the role of primary care includes prescribing, monitoring and supporting de-prescribing. ${ }^{23}$ The Quality and Outcomes Framework, an annual incentive scheme for all general practices in England, often dictates what monitoring is required. These monitoring indicators are quite broad, including four mental health indicators, for example: "The percentage of patients with schizophrenia, bipolar affective disorder and other psychoses who have a record of BMI [body mass index] in the preceding 12 months". These targets should not just be seen as a 'tick-box' duty but, as pointed out by the complexities described previously, should be taken as an opportunity to intervene and improve care for individual patients when problems or abnormalities are identified. Care could be improved if there were stronger links between specialists (including psychiatrists, mental health nurses and pharmacy staff) and primary care. ${ }^{24}$ The recent Carter report on mental health services in England concluded that specialist mental health pharmacy is a 
critical clinical service that is key in ensuring high-quality patient care. ${ }^{12}$ Carter highlighted the need for specialists to move outside of their in-patient units and support patients in the community, thereby suggesting intensified collaboration with GPs.

Medication is one of the key treatments for people with mental health problems. Medication optimisation for people with SMI requires a multidisciplinary approach putting the person with SMI at the centre. Healthcare professionals need to work together with patients and family carers to individualise care and treatment choices, monitor outcomes more carefully, review medicines more frequently and provide support to people with SMI when needed. The indicators described by Khawagi et al are a helpful addition to monitoring safety and identifying areas for improvement at a local, regional and national level.

Correction notice The paper has been corrected since it published online. Co-author's name amended from Caroline Chew-Graham to Carolyn Chew-Graham.

Twitter Ian Maidment @maidment_dr

Funding The authors have not declared a specific grant for this research from any funding agency in the public, commercial or not-for-profit sectors.

Competing interests None declared.

Patient consent for publication Not applicable.

Provenance and peer review Commissioned; internally peer reviewed.

ORCID iD

Ian Maidment http://orcid.org/0000-0003-4152-9704

\section{REFERENCES}

1 Centre For Mental Health (for NHS), Taskforce MH. The five year forward view for mental health. The Mental Health Taskforce, 2016.

2 Ghebreyesus T. The WHO Special Initiative for Mental Health (2019-2023). WHO, 2019. Available: https://www.who.int/ publications/i/item/special-initiative-for-mental-health-(2019. 2023)

3 Carr MJ, Steeg S, Webb RT, et al. Effects of the COVID-19 pandemic on primary care-recorded mental illness and selfharm episodes in the UK: a population-based cohort study. Lancet Public Health 2021;6:e124-35.

4 Naylor C, Bell A, Baird B. Mental health and primary care networks: understanding the opportunities, 2020. Available: https://www.kingsfund.org.uk/publications/mental-healthprimary-care-networks

5 Cooper SJ, Reynolds GP, et al, With expert co-authors (in alphabetical order). BAP guidelines on the management of weight gain, metabolic disturbances and cardiovascular risk associated with psychosis and antipsychotic drug treatment. $J$ Psychopharmacol 2016;30:717-48.

6 Maidment ID, Lelliott P, Paton C. Medication errors in mental healthcare: a systematic review. Qual Saf Health Care 2006;15:409-13.

7 WHO. World Health Organisation. Addressing comorbidity between mental disorders and major noncommunicable disease, 2017. Available: https://www.euro.who.int/_data/ assets/pdf_file/0009/342297/Comorbidity-report_E-web.pdf
8 Paton C, Esop R, Young C, et al. Obesity, dyslipidaemias and smoking in an inpatient population treated with antipsychotic drugs. Acta Psychiatr Scand 2004;110:299-305.

9 Roberts L, Roalfe A, Wilson S, et al. Physical health care of patients with schizophrenia in primary care: a comparative study. Fam Pract 2007;24:34-40.

10 Khawagi WY, Steinke D, Carr MJ, et al. Evaluating the safety of mental health-related prescribing in UK primary care: a cross-sectional study using the clinical practice research Datalink (CPRD). BMJ Qual Saf 2021. doi:10.1136/ bmjqs-2021-013427. [Epub ahead of print: 25 Aug 2021].

11 Khawagi WY, Steinke DT, Nguyen J, et al. Development of prescribing safety indicators related to mental health disorders and medications: modified e-Delphi study. Br J Clin Pharmacol 2021;87:189-209.

12 Carter. NHS operational productivity: unwarranted variations mental health services and community health services, 2018. Available: https://improvement.nhs.uk/about-us/corporatepublications/publications/lord-carters-review-unwarrantedvariations-mental-health-and-community-health-services/

13 NICE. Medicines optimisation: the safe and effective use of medicines to enable the best possible outcomes. NICE guidelines, 2015. Available: www.nice.org.uk/guidance/ng5/ resources/medicines-optimisation-the-safe-and-effective-use-ofmedicines-to-enable-the-best-possible-outcomes-51041805253 [Accessed 12 Oct 2016].

14 Mangin D, Bahat G, Golomb BA, et al. International Group for Reducing Inappropriate Medication Use \& Polypharmacy (IGRIMUP): Position Statement and 10 Recommendations for Action. Drugs Aging 2018;35:575-87.

15 Kadra G, Stewart R, Shetty H, et al. Long-term antipsychotic polypharmacy prescribing in secondary mental health care and the risk of mortality. Acta Psychiatr Scand 2018;138:123-32.

16 Galbally M, Frayne J, Watson SJ, et al. Psychopharmacological prescribing practices in pregnancy for women with severe mental illness: a multicentre study. Eur Neuropsychopharmacol 2019;29:57-65.

17 Whicher CA, Price HC, Holt RIG. Mechanisms in endocrinology: antipsychotic medication and type 2 diabetes and impaired glucose regulation. Eur J Endocrinol 2018;178:R245-58.

18 Houben N, Janssen EPCJ, Hendriks MRC, et al. Physical health status of older adults with severe mental illness: the PHiSMI-E cohort study. Int J Ment Health Nurs 2019;28:457-67.

19 Vinogradova Y, Coupland C, Hippisley-Cox J, et al. Effects of severe mental illness on survival of people with diabetes. Br J Psychiatry 2010;197:272-7.

20 Becker T, Hux J. Risk of acute complications of diabetes among people with schizophrenia in Ontario, Canada. Diabetes Care 2011;34:398-402.

21 Fox C, Richardson K, Maidment ID, et al. Anticholinergic medication use and cognitive impairment in the older population: the Medical Research Council cognitive function and ageing study. J Am Geriatr Soc 2011;59:1477-83.

22 Bignall T, Jeraj S, Helsby E. Racial disparities in mental health: literature and evidence review. race equal found heal wellbeing alliance 2019:60.

23 Newbigging K, Durcan G, Ince R, et al. Filling the chasm. Centre for Mental Health, 2018.

24 NHS England. Five year forward view for mental health: one year on. England: NHS, 2017. https://www.england.nhs.uk/ wp-content/uploads/2017/03/fyfv-mh-one-year-on.pdf 